\title{
Los desafíos de la transformación digital de la democracia ${ }^{1}$
}

\section{The Challenges of the Digital Transformation of Democracy}

\author{
JORGE FRANCISCO AGUIRRE SALA (UNIVERSIDAD AUTÓNOMA DE NUEVO LEÓN - \\ INSTITUTO DE INVESTIGACIONES SOCIALES)
}

Artículo recibido: 8 de septiembre de 2020

Solicitud de revisión: 26 de febrero de 2021

Artículo aceptado: 27 de marzo de 2021

\begin{abstract}
Aguirre Sala, Jorge Francisco (2021). Los desafíos de la transformación digital de la democracia. Recerca. Revista de Pensament i Análisi, 26(2), pp. 1-23.

doi: http://dx.doi.org/10.6035/recerca.466o
\end{abstract}

\section{Resumen}

Las tecnologías digitales provocaron la expectativa de mejorar la democracia al generar espacios e instrumentos para incrementar la participación ciudadana. Sin embargo, algunos de sus usos han inducido a denigrar la deliberación democrática. Con un método analítico aplicado a las referencias teóricas y los resultados de investigaciones empíricas, se revisan los desafíos democráticos de las tecnologías digitales para mostrar su índole política y ética. Los resultados muestran razones para abandonar el optimista tecnodeterminismo. Las conclusiones sugieren, por una parte, que la tecnología digital puede resolver los obstáculos de la sofisticada información sobre políticas públicas y la complejidad del enorme número de participantes. Pero, por otro lado, no se deben homogeneizar los procesos digitales, ni encasillarlos en modelos de democracia electrónica, y los defectos endógenos de la tecnología se deben inhibir con diseños autocorrectivos. La tarea de las tecnologías digitales a futuro estriba en desarrollar la inteligencia artificial para instrumentar el mayor número posible de mecanismos de participación ciudadana, ampliar su alcance demográfico para abatir las brechas, complementar los procedimientos de mediación y promover la deliberación incluyente. El corolario muestra que el futuro de la democracia electrónica está en la pedagogía tecnopolítica.

Palabras clave: noticias falsas, algoritmos, vigilancia, control, polarización, oligopolio.

\footnotetext{
Este texto forma parte del proyecto de investigación sobre democracia electrónica, financiado por el Programa de Apoyo a la Investigación Científica y Tecnológica 2020, clave CSA 1523-20 de la Universidad Autónoma de Nuevo León, México. El autor, líder del cuerpo académico «Democracia y Sustentabilidad», agradece a la Secretaría y a la Dirección de Investigación Científica y Desarrollo Tecnológico de la UANL el financiamiento recibido.
} 


\section{Abstract}

Digital technologies raised the expectation of improving democracy by generating spaces and instruments to increase citizen participation. However, some of its uses have led to denigrate democratic deliberation. With an analytical method on theoretical references and the results of empirical research, the democratic challenges of digital technologies are reviewed to show their political and ethical nature. The results show reasons to abandon optimistic techno-determinism. The findings suggest, on the one hand, that digital technology can solve the obstacles of sophisticated public policy information and the complexity of the huge number of participants. But, on the other hand, digital processes should not be homogenized, nor pigeonholed into models of electronic democracy; endogenous defects should be inhibited with self-correcting designs. The task of digital technologies in the future is to develop artificial intelligence to implement the largest possible number of citizen participation mechanisms, expand their demographic reach to close gaps, complement mediation procedures and promote inclusive deliberation. The corollary shows that the future of electronic democracy lies in techno-political pedagogy.

Key Words: fake news, algorithms, surveillance, control, polarization, oligopoly.

\section{INTRODUCCIÓN}

La tecnología digital, en tanto ícono de progreso, al usarse en el ámbito político también provocó la optimista expectativa de que mejoraría la democracia. Sin embargo, al revisar aspectos estructurales de la democracia y no solo incidencias en momentos coyunturales, el optimista ánimo tecnodeterminista fue deteriorándose. Incluso se piensa que las tecnologías digitales, en vez de mejorar la democracia, pueden empeorarla.

El propósito de este texto es mostrar que algunos desafíos y riesgos de la democracia electrónica son éticos y políticos. Porque las tecnologías digitales, si bien aportan logros en el sentido de superar déficits de tipo procedimental, desde la perspectiva ética y política abren la posibilidad a acciones antidemocráticas.

Para explicar lo anterior, se procede con un método analítico aplicado a las referencias teóricas y los resultados de investigaciones empíricas que permite desarrollar las siguientes secciones: 1) descripción de la democracia electrónica para distinguirla de los usos que las tecnologías digitales tienen en el gobierno electrónico, 2) análisis de los desafíos éticos y políticos que enfrenta la democracia debido a las tecnologías digitales, 3) las razones de estudiosos teóricos para abandonar el ciberoptimismo, 4 ) algunos datos empíricos desilusionantes y 5 ) conclusiones. 


\section{DEMOCRACIA ELECTRÓNICA}

A principios de los años noventa del siglo XX emergió la noción de ciberdemocracia ligada al concepto de ciberespacio (Rheingold, 1993). La ciberdemocracia abogó por la democracia directa y la idea de un espacio sin espacio, es decir, un lugar virtual. Provocó una discusión que cuestionaría la territorialidad como limitante a los ciudadanos, es decir, la restricción a poseer representantes políticos solo por la circunscripción del domicilio.

La ciberdemocracia desarrolló sus postulados en las versiones de Hagen (1997) y Bellamy (2000): modalidad directa, visión antiestatal o de control gubernamental minimalista, omisión de una identidad ciudadana fija que permite asumir tantos roles como las tecnologías digitales lo permitan, promoción del activismo y restablecimiento de la autonomía en la sociedad civil. La ciberdemocracia constituye un modelo prescriptivo y normativo y, por esto, se distingue de la democracia electrónica; esta última nació con la paulatina incorporación de las incipientes tecnologías de comunicación electrónicas, y después las digitales, a los procesos democráticos que existían previamente. Por eso, autores como Vedel (2003) registran sus antecedentes desde 1950.

En la actualidad se aluden dos definiciones para la democracia electrónica: la del Consejo de Ministros del Consejo de Europa y la de Trechsel, Kies, Mendez y Schmitter. La definición de estos autores reza:

La e-democracia consiste en todos los medios electrónicos de comunicación que habiliten/ayuden a los ciudadanos en sus esfuerzos por supervisar y controlar gobernantes/políticos sobre sus acciones en el poder público. Dependiendo de qué aspecto democrático se esté promoviendo, democracia y electrónica puede emplear diferentes técnicas: (1) mejorar la transparencia del proceso político; (2) para facilitar la participación directa y la participación de los ciudadanos; y (3) para mejorar la calidad de la formación de opinión mediante la apertura de nuevos espacios de trabajo (Trechsel, Kies, Mendez y Schmitter, 2004: 3).

La Unión Europea, a través del Consejo de Ministros del Consejo de Europa, considera que la democracia electrónica es el:

[...] apoyo y fortalecimiento de la democracia, de las instituciones democráticas y los procesos democráticos por medio de las TIC [...] una oportunidad para permitir y facilitar el suministro de información y deliberación, fomentar la participación ciudadana con el fin de ampliar el debate político, y favorecer una mejor y más legítima adopción de decisiones políticas (Comité de Ministros del Consejo de Europa, 2009: 4-5) 
El Consejo de Ministros realiza doce recomendaciones para aprovechar la democracia electrónica a favor del fortalecimiento de la democracia en sus instituciones y procesos, así como la vinculación de la participación ciudadana (Comité de Ministros del Consejo de Europa, 2009: 5-7). En el apéndice de su declaración incluyó 80 principios y 102 directrices. Las anteriores definiciones y caracterizaciones muestran, como se ha dicho, la paulatina incorporación de las tecnologías a los procesos democráticos que existían previamente.

La definición del Consejo de Ministros del Consejo de Europa tiende hacia avances democratizadores dentro de los marcos jurídicos previamente institucionalizados. Mientras que la definición de Trechsel, Kies, Mendez y Schmitter, cuatro años antes, se inclina más por fortalecer la soberanía de cada ciudadano en vez de robustecer modelos democráticos ya establecidos. Su propensión apunta hacia la perspectiva ascendente y ciudadanizada; no considera la democracia electrónica desde arriba, para evitar la simple réplica del modelo representativo ejercido por tecnologías digitales. Luego entonces, parece que no se trata de adoptar una u otra posición entre desde arriba o abajo, ciberoptimismo o ciberpesimismo, sino de comprender la democracia más centrada en los ciudadanos y no tanto en las instituciones que instrumentalizan digitalmente sus procesos con el propósito de legitimar sus procedimientos.

La democracia electrónica se distingue del gobierno electrónico porque es más vasta que la administración digital de los servicios públicos estatales. Porrúa, al citar al Grupo de Trabajo sobre Gobierno Electrónico en países en desarrollo, advierte: "computadoras no es lo mismo que reformas y que la "e" del e-government se refiere solo a una herramienta para construir lo que algunos expertos denominan el i-government o gobierno inteligente» (Porrúa, 2004: 3). De similar forma lo señalaron Abramson y Means respecto a los planes de gobierno electrónico: «deben pensar en términos de transformación de procesos y no solo de automatización de los mismos» (Abramson y Means, 2001: 3). La evolución de la democracia electrónica apunta a la definición, diseño, planeación, programación y evaluación de las decisiones del Estado en ámbito de las políticas públicas.

El gobierno electrónico se focaliza en la digitalización instrumental de la administración estatal, pero no incide en la definición de las políticas públicas a menos que evolucione hacia un modelo de gobierno abierto o gobernanza. Por ejemplo, no es lo mismo pagar impuestos a través de instrumentos digitales que deliberar y definir la razón, el monto y el destino de las cargas tributarias. Al inicio del gobierno electrónico de generó optimismo por la sensación de transparencia, pero con el tiempo surgen problemas de seguridad, malos 
contenidos, correo electrónico no deseado, virus y robos de identidades. Estos inconvenientes, por ser comunes al gobierno y la democracia electrónicos también provocan su equiparación.

La democracia electrónica tampoco ha de confundirse con el ciberactivismo político que aborda problemas circunstanciales, porque no es la movilización de protesta. Si bien busca influir en el Estado, lo hace con miras a transformarlo estructuralmente para luego facilitar la tarea democrática a gobernados y gobernantes. Es decir, persigue la institucionalización, el reconocimiento jurídico y la ejecución de variados instrumentos participativos, en vez de correcciones contingentes. En consecuencia, la democracia electrónica, en cuanto implementa la digitalización de mecanismos emergentes de participación, posee puntos de intersección con el gobierno abierto y la gobernanza.

\section{LOS PERJUICIOS DEL USO DE LAS TECNOLOGÍAS DIGITALES PARA LA DEMOCRACIA}

Entre los desafíos éticos más comunes están la falsedad y la saturación de información con intenciones dolosas. Ambas debilitan la capacidad cognitiva, opacan la transparencia y promueven emociones en vez de construir la deliberación democrática necesaria para el voto, iniciativas legislativas, presupuestos participativos, referéndums, etc. Con una mezcla de posverdad e infointoxicación, el ciudadano común podría confundir la realidad virtual y la vida real colectiva. Esta indistinción es denunciada por Calvo: «[...] confunde la identidad de un significado con la identidad de un objeto» (Calvo, 2019b: 682); de manera que puede provocar que la participación en la red digital carezca de efectos reales en política, al grado que Morozov advierte: «el cloud activismo desprecia otras prácticas democratizadoras» (Morozov, 2011: 170).

Quizá lo peor de la información falsa es la misma performatividad de las redes sociales: existe gran equivocidad al equiparar un retuit en Twitter con un voto electrónico o de confianza, o una suscripción en Youtube con apoyar una petición electrónica. El debate y los seguimientos en las redes sociales no son equivalentes a la democracia asamblearia o las adhesiones. La misma performatividad y mercadotecnia de las redes sociales seduce hacia la falacia de equiparar el número de seguidores con la investidura de representatividad (Cha, Haddadi, Benevenuto y Gummad, 2010; Caldevilla, 2009). La cultura digital ha explotado la cultura del narcisismo, es decir, el objetivo de los usuarios es conseguir seguidores y buenas evaluaciones en las audiencias digitales 
(Keen, 2012), en vez de compartir propuestas, ricas en contenidos y alcances demográficos.

Otra faceta sutil de la información falsa consiste en los impostores del activismo de base (conocido en inglés como astroturfing). Consiste en la promoción, deliberadamente disfrazada, de un movimiento social que aparenta ser genuino y cabalmente ciudadano, cuando en realidad fue planeado y desarrollado por grupos de interés. La información falsa o saturante, el ciberactivismo que no persigue transformaciones estructurales y la impostura del activismo de base dan por resultado una esfera pública virtual que sustituye y erosiona la soberanía del ciudadano común, sin llegar a mejorar el ejercicio de la democracia.

La democracia electrónica, sobre todo por su necesidad de deliberación, también se degrada por la vigilancia y la violación de la privacidad digital a través de los registros individuales o masivos que las tecnologías digitales efectúan.

Por un lado, se trata de la información controlada que los buscadores electrónicos arrojan a los ciudadanos gracias a los algoritmos. El manejo de datos a gran escala detecta preferencias para manipular a los usuarios, pues los algoritmos provocan el efecto burbuja (Pariser y Helsper, 2011), es decir, la cooptación de electores en las esferas informativas afines a su ideología que evita el contraste de ideas y acaba con la deliberación. El efecto burbuja trastorna la transparencia requerida en la rendición de cuentas gubernamental y la validación de las auditorias ciudadanas. A su vez, provoca los sesgos de las cámaras eco, es decir, la repetición y recepción acrítica de los mensajes que intentan modular las actitudes políticas de los ciudadanos. Una secuela palpable de los algoritmos es la exposición selectiva para establecer en los usuarios una percepción temática que determine la agenda pública y, en consecuencia, la agenda política (acción denominada en inglés como agenda setting).

Las cámaras eco, al provocar el aislamiento de otras fuentes informativas, también estimulan la polarización (Boutyline y Willer, 2017; Flaxman, Goel y Rao, 2016). Al ciudadano se le acostumbra a navegar solo por las redes que coinciden con sus pareceres, entonces la información fluye en contenidos con espirales extremistas que fragmentan las comunidades (fenómeno también descrito como Cyberbalkanization por Van Alstyne y Brynjolfsson, 2005) y derivan en el discurso del odio. Hay un uso perverso de las tecnologías digitales que genera estratos confinados y polarizados intencionalmente con hiperatención selectiva (llamado Ghettoization por Wright y Jacobs, 1994). 
Por otra parte, se trata de la violación de la privacidad que ha tomado la modalidad llamada capitalismo de vigilancia (Zuboff, 2019) o internet de las cosas. Consiste en aprovechar la hiperconectividad digital que los ciudadanos tienen (muchas veces, involuntariamente) a instancias privadas y públicas a través de sus dispositivos digitales. Por ejemplo, el pretendido control de la pandemia COVID-19 en varios países utilizó algoritmos con la información de ubicación, tránsito, reunión y reconocimientos biométricos provenientes no solo de cámaras digitales en zonas públicas, sino de la obligación impuesta a los ciudadanos a utilizar códigos y señales que emiten los dispositivos digitales. Esta tecnocracia digitalizada (con independencia de su efectividad sanitaria) no representa ninguna práctica democrática. En el extremo de la vigilancia se utilizan softwares de espionaje como FinFisher, Galileo y Pegasus con capacidad para grabar conversaciones, descargar archivos, listas de contactos y mensajes de texto guardados en los dispositivos; inclusive, pueden activar la cámara y micrófono del equipo invadido.

El reto ético consiste en desenmascarar la estratagema política: «El poder no lo tienen los medios [...] son el espacio donde se crea el poder» (Castells, 2009: 262), con el propósito de dominar a sus usuarios. Los medios se usan para producir la ilusión de una libertad política. Así, la política tradicional somete a los usuarios de las tecnologías digitales sin su apercibimiento. Kosinski, uno de los creadores del algoritmo utilizado por Cambridge Analytica para manipular la elección presidencial de EE. UU. en el 2016, explica cómo en la era digital «si una persona no paga por un producto o servicio es porque la persona es el producto» (Kosinski, 2017: 4). Es decir, al usuario se le convierte en potencial consumidor, se le cosifica moldeándolo como la demanda dentro de la fórmula mercantil de la oferta y la demanda. Cuando un usuario acepta, por ejemplo, una cuenta gratuita de correo electrónico o de Instagram, queda convertido en el potencial consumidor de las ofertas de consumo que por esas vías se le ofrecerán.

En el aspecto electoral, la comodidad de las aplicaciones digitales moldea en el usuario-ciudadano la falta de criticidad. En varios países (como Suecia, Alemania, Francia, Finlandia, Austria y España) aplicaciones digitales facilitan la decisión electoral de acuerdo con el perfil del votante. Por ejemplo, VoteSwiper (https://www.voteswiper.org/es) y Tuvoto.eu son aplicaciones que garantizan por cuál partido político inclinarse, de acuerdo con el perfil personal elaborado previamente con un cuestionario. Es decir, las elecciones de representantes políticos se llevan a cabo por servicios digitales sin oportunidad de discernimiento, ni interacción con los candidatos. Cerrando el círculo 
electoral, las tecnologías digitales también pueden suplantar a los representantes construyendo «políticos algorítmicos» (Calvo, 2019a: 15), es decir, postular robots como candidatos. So pretexto de que los algoritmos podrán tomar decisiones públicas sin contaminación emocional, corrupción o falta de profesionalidad, las tecnologías digitales tienden a «la sustitución de personas por modelos matemáticos en los gobiernos democráticos» (Calvo, 2019a: 13).

En sentido contrario, si se desea evitar la despersonalización, entonces surge el desafío ético ante la información y la propaganda masiva que promueven la concepción de la política como una expresión de sentimientos y no como ámbito de confrontación de ideas y programas, lo que también es aprovechado para nutrir al populismo y anular la deliberación. De este modo, un gran reto social estriba en regular la veracidad y las incidencias psicológicas en uso de las tecnologías digitales.

En la era digital, la lucha por el espacio público virtual y el reconocimiento sobre su dominio no surgió normada con cláusulas constitucionales, mandatos jurídicos o sentencias judiciales sobre los alcances de las tecnologías digitales. Simplemente los códigos digitales funcionaron como ley (Lessig, 1999) y tal parece que así han continuado, al grado que Berners-Lee, considerado el creador de la red, solicitó a los gobiernos, las empresas y la ciudadanía que suscribieran El contrato para la Web (2019).

En el tenor de lo anterior, otro reto ético en el uso de las tecnologías digitales consiste en no reproducir la división de clases sociales y políticas con su consecuente inequitativa distribución del poder. La brecha digital no solo es consecuencia de la desigualdad social, sino que, a su vez, también la conserva y la provoca. Por ende, el poder de la ciudadanía decrece. Howard et al. (2016), en un estudio de circulación internacional sobre el compromiso cívico y el clickactivismo en el caso mexicano de «El Bronco» (el sobrenombre del político que ganó una elección realizando su campaña electoral centrado en las redes sociales), describen cómo el uso de las plataformas digitales se inicia como foros de posicionamiento político y se convierte en espacios de quejas y exigencias de servicios públicos; o cómo las tecnologías digitales logran altos grados de expresión o cohesión entre oprimidos, pero no alcanzan compromisos sustantivos o influencia real para establecer la agenda pública.

El debilitamiento de las mediaciones institucionales atingentes a partidos políticos, legislaturas y organismos públicos autónomos es un perjuicio más de las tecnologías digitales en la política. El poder de la opinión pública corre el riesgo de proceder sin mediaciones, ni depuración informativa, por los ataques hacker con softwares automatizados que operan como robots o los vigilantes 
humanos que desean trastornar emotivamente a los participantes para que muden sus actitudes y peticiones políticas. De ello se sigue la ruptura comunicativa que implica una ruptura institucional y, por ende, comunitaria. Cotarelo (2002) advirtió que la democracia electrónica atenta contra la reflexión deliberativa debido a su comunicación directa, de inmediatez e instantaneidad. Pues viralizar un asunto local en el ámbito nacional para sacarlo de proporción o destruir una figura pública son acciones propias del activismo y no de la democratización de una sociedad. Es necesario «Distinguir entre la función crítica y desestabilizadora de internet y la capacidad de construcción democrática» (Innerarity, 2015: 17), porque la experiencia muestra que: «Internet facilita la destrucción de regímenes autoritarios, pero no es eficaz para consolidar la democracia» (Innerarity, 2015: 43).

Por lo que corresponde a los gobiernos, desde 1993 Rheingold advirtió que las tecnologías digitales serían una nueva versión de la «razón instrumentalizada», es decir, medios mercantilizados al servicio del poder hegemónico que no tiene intenciones democratizadoras. Las tecnologías son instrumentos que, a su vez, son instrumentalizados para ofrecer la sensación de transparencia y legitimidad. Con ello opacan a otros agentes sociales que desean intervenir en los procesos políticos e, inclusive, los enfrenta con otros mecanismos de democracia participativa (plebiscitos, consultas, peticiones, revocatorias). Las tecnologías digitales, al ser cosificadas, fueron reducidas a utensilios de interés de clase.

A lo anterior se suma una nueva élite política con el oligopolio de internet. El riesgo añade el peligro de abandonar las tecnologías digitales en manos de quienes tienen el control sobre la banda ancha para vigilar, desinformar e imponer la colonización cultural. Las tecnologías digitales quedan subordinadas al monopolio privado de portales controlados por sus propietarios. Esto se extendió hasta acuñar el término Googlearchy (Van Djik y Hacker, 2018: 39 y ss.) y se reconoce que Google, Amazon, Facebook, Apple y Microsoft constituyen el monopolio de las tendencias culturales, las futuras formas de vida y, por ende, de las directrices políticas (Smyrnaios, 2016). El oligopolio de internet norma y normaliza las tecnologías digitales a modelos democráticos verticales o francamente antidemocráticos.

García advierte que las evaluaciones de las tecnologías digitales respecto a la democratización «siempre dependerán de la adopción de una determinada concepción de la democracia» (García, 2016: 172). En consecuencia, las tecnologías digitales no pueden determinar un tipo de organización democrática, sino que las modalidades de la política determinan el rol de las tecnologías 
digitales dentro de una tradición política. Esto explica por qué los resultados empíricos sobre el éxito de la democracia electrónica cambian de un país a otro. Por tanto, no es procedente juzgar la democracia electrónica, ni las tecnologías digitales, conforme a modelos democráticos. Las prácticas de democracia electrónica y los planificadores teóricos no han logrado un modelo democrático idóneo para las tecnologías digitales, hasta el grado de que Päivärinta y Saebo reconocieron una «jungla» (2006: 818) de modelos a la democracia electrónica imposibles de conciliar. Lindner y Aichholzer ratifican que no es posible un modelo universal de democracia electrónica: «Podría decirse que las preferencias por un determinado modelo de democracia probablemente determinarán el tipo de la democracia electrónica que un proponente busca establecer» (Lindner y Aichholzer, 2020: 15). ${ }^{2}$

El propio Comité de Ministros del Consejo de Europa reconoce que la democracia electrónica depende del desarrollo que haya tenido hasta el momento la democracia moderna (2009: 49). Si en esta se han adaptado convenientemente las tecnologías digitales, entonces la tecnología solo instrumentaliza el éxito democratizador, pero por sí misma la democracia electrónica no conduce a más y mejor democracia. El optimismo afronta que la esfera pública digitalizada no se ha convertido en más igualitaria, a pesar de muchas iniciativas institucionales para lograrlo (Chadwick y Howard, 2009; Moss y Coleman, 2014).

En lo concerniente a la representatividad, existen instituciones políticas con portales web muy elaborados. Sin embargo, no siempre significa que sean efectivamente abiertas o inclusivas. Aguirre y Jones (2012), Trechsel, Kies, Mendez y Schmitter (2004) y Ricci (2013) aportan análisis de miles de sitios web de parlamentos, partidos y representantes políticos de todas latitudes. Los resultados son similares: los actores políticos casi siempre proceden de manera informativa, unilateral, sin potencial interactivo ni efectos deliberativos o vinculantes. Tal parece que la democracia electrónica carece de un programa de transición, pues los actores políticos tradicionales conservan sus defectos.

En una primera conclusión provisional, puede decirse que las tecnologías digitales tienen algunos efectos poco alentadores para la democracia. Parecería que poner las tecnologías digitales en la política tiene el mismo riesgo que poner tecnologías nuevas al alcance de añejos vicios políticos. 


\section{LAS RAZONES DE ESTUDIOSOS TEÓRICOS PARA ABANDONAR EL CIBEROPTIMISMO}

En un texto de estas dimensiones es imposible recapitular todos los análisis acerca de la incursión democrática que han tenido las tecnologías digitales. Según Ricci, entre el año 1995 y 2005, el término democracia electrónica —sin considerar sus variados sinónimos- se ha utilizado en casi 1600 artículos académicos y en 2330 ooo páginas web (Ricci, 2013: 36). Hennen et al. indagaron solo entre los años 2011 y 2016 y seleccionaron 3600 referencias significativas; además, mediante varios pasos de filtrado, redujeron la literatura principal más relevante a alrededor de 400 títulos (Hennen et al., 2020: 6). Aunque es un riesgo seleccionar y canonizar algunas investigaciones, no se pueden ignorar los hallazgos de las más destacadas, entre otras, las realizadas por Hindman, Van Dijk y Hacker, Castells, Barber o Runciman.

Las investigaciones de Hindman (2009) son valorizadas como un referente. Mostró razones permanentes de por qué internet no logra cambiar los regímenes constitucionales no democráticos en regímenes democráticos. Las tecnologías digitales solo logran incrementar algunos elementos del proceso de democratización. El listado de esos elementos puede obtenerse del mapa elaborado por Krimmer (2009) y podría conjeturarse que, de los 35 elementos que constituyen el mapa de la moderna democracia electrónica, no todos poseen el mismo nivel evolutivo gracias a las tecnologías digitales. En particular, los más renuentes y conflictivos son aquellos que requieren de deliberación, logro de consensos, capacitación y definición unificada de políticas públicas.

Hindman advierte respecto a la deliberación democrática que: «la esfera pública en el ciberespacio podría ser algo cercano al ideal Habermasiano, pero como Chadwick [(2006: 102)] ha dicho "El camino hacia la democracia electrónica está plagado de los cascos quemados de proyectos fallidos"» (Hindman, 2009: 138), dado que «La esfera pública en línea ya es una aristocracia de facto dominada por los expertos en las altas artes deliberativas» (Hindman, 2009: 139). De manera que la democracia digital es la mezcla de nuevas tecnologías y viejos fracasos (Hindman, 2009: 141 y ss.). Poner las tecnologías digitales en la política, como lo muestra la primera conclusión provisional, tiene el mismo riesgo que poner el vino nuevo en odres viejos.

Uno de los seguimientos históricos más reconocidos de la pretendida transformación democrática por la cultura digital se debe a Van Dijk y Hacker. Desde el año 200o, con la aparición del texto Digital Democracy, hasta el año 2018, con la publicación de Internet and Democracy in the Network Society, 
ambos autores han revisado la evolución de la democracia electrónica. Después de casi veinte años de indagaciones, exponen razones para «abandonar el ciberoptimismo» (Van Dijk y Hacker, 2018: xi). Entre las principales razones se encuentran que la automatización de la democracia y el uso de los new media simplemente han sido extensiones de lo que ya ocurría con la comunicación e interacción política fuera de las tecnologías digitales (Van Dijk y Hacker, 2018: 13). Que ante la expectativa de que el uso de las tecnologías digitales realmente cambiara las estructuras de los sistemas políticos y democráticos, hallaron el principio de que la política es lo primero y los medios están en segundo lugar (Van Dijk y Hacker, 2018: 19). Es decir, que la política debe primero ser democrática para que las tecnologías digitales y sus funciones también lo sean. Y ello porque «Internet en sí mismo no es más democrático que los antiguos mass media» (Van Dijk y Hacker, 2018: 47). Que la participación política en línea también puede ser antidemocrática y que no tiene efectos en el sistema político de una nación (Van Dijk y Hacker, 2018: 53). Que cambiar un sistema político es mucho más difícil que organizar por internet organizaciones políticas comprometidas (Van Dijk y Hacker, 2018: 94). Que las acciones deliberativas online difícilmente llegan a resultar en consensos o conclusiones, al tiempo que los representantes políticos y los gobernantes no aceptan los resultados de la participación electrónica (Van Dijk y Hacker, 2018: 103). Que después de experimentar durante cinco años los resultados estructurales que siguieron a la Primavera Árabe, estos fueron más negativos que positivos (Van Dijk y Hacker, 2018: 145). Que, como también lo indican Innerarity (2015: 43) y Bellin (2012: 139), los new media son útiles para derrocar las dictaduras, pero no son eficaces para construir una nación democrática (Van Dijk y Hacker, 2018: 147). Que la satisfacción ciudadana por un partido o candidato que alcanzó el poder por las tecnologías digitales solo significa que son ganadores, no necesariamente democráticos, a menos que habiliten cambios democráticos como un sistema de gobernanza (Van Dijk y Hacker, 2018: 193). Que el caso de China muestra a las tecnologías digitales apoyando más el autoritarismo que la emergencia de la democratización (Van Dijk y Hacker, 2018: 199). Que internet no ha cambiado las reglas del constitucionalismo, ni las leyes que regulan el gobierno, ni los sistemas electorales. De manera que la única transformación que se observa es el tránsito de la participación offline a la online, sin resultados en los cambios estructurales de los sistemas políticos (Van Dijk y Hacker, 2018: 205). Finalmente, también señalan que «no esperan que el uso del big data y la inteligencia artificial para la participación electrónica sea una solución para 
las fallas democráticas» (Van Dijk y Hacker, 2018: 210). En pocas palabras, una y otra vez sus hallazgos empíricos los llevan a concluir que la política va primero y las tecnologías digitales vendrán detrás (Van Dijk y Hacker, 2018: 19, 52-53, 77, 94, 147, 198, 206).

Pero no solo Van Dijk y Hacker llegar a esta conclusión. Castells reconoció desde inicio del siglo XXI que «las Tecnologías de Información y Comunicación no han cumplido la utopía democrática» (Castells, 2001: 177). Trechsel, Kies, Mendez y Schmitter, al evaluar los cambios históricos dentro de la democracia, consideran que, a pesar de las transformaciones de los mecanismos, los principios centrales no se inmutan y, por lo tanto, «nuestra expectativa a largo plazo debería ser que las tecnologías digitales no alterarán fundamentalmente la naturaleza de la democracia» (Trechsel, Kies, Mendez y Schmitter, 2004: 2). Runciman, comparando la teoría política y la real politik, tres lustros después, asevera que «La política tiene sus propios imperativos y no marchará al ritmo del tambor tecnológico» (Runciman, 2017: 14). Barber, en alusión a la política norteamericana, también advirtió en un sentido casi idéntico que «Es la política quien hará democrática a la tecnología. La tecnología no hará democrática a la política. La política es lo primero por lo que hay que luchar para conseguir una tecnología democrática» (Barber, 2006: 26). Lidén, basado en sus estudios sobre la sociedad sueca, también concluyó que «la democracia electrónica no puede ser por sí misma totalmente democrática si el sistema político en el que está incrustada no es democrático» (Lidén, 2012: 85).

Ante estas conclusiones, algunos datos empíricos verifican que las tecnologías digitales no proporcionan aportes de igual importancia a todos los elementos de la democracia. En efecto, el optimismo político generado por la transformación digital solo alcanza buenos «momentos democráticos» (Levitsky y Ahmad, 2015: 53-58).

\section{ALGUNOS DATOS EMPÍRICOS DESILUSIONANTES}

Al referirse a datos empíricos sobre las experiencias de la democracia electrónica, generalmente se hace alusión a las denominadas mejores prácticas o prácticas exitosas. En este contexto no pueden desconocerse los casos paradigmáticos recabados, por encomienda del Parlamento Europeo, a través de su Panel for the Future of Science and Technology (STOA), por Hennen et al. (2020). Tampoco pueden ignorarse las experiencias destacadas por Simon, Bass, Boelman y Mulgan (2017), por encomienda de NESTA y MacArthur 
Foundation Research Network on Opening Governance. Aunque los casos de prácticas exitosas de democracia electrónica son variados, parece existir un consenso de los más logrados: peticiones electrónicas, uso de tecnologías digitales por partidos políticos y gobiernos locales y algunas acciones digitales significativas en tareas parlamentarias.

Sin embargo, otros estudios empíricos muestran conclusiones ambivalentes. Por ejemplo, Anduiza, Jensen y Jorba (2012), en quince casos de diversos países, muestran que las tecnologías digitales desarrollan mecanismos y vías de participación ciudadana que aumentan el compromiso político. Pero, ante esos datos contrastan las treinta y seis investigaciones de Boulianne (2015). Los resultados muestran que el $50 \%$ los coeficientes entre el uso de las redes sociales y la participación en la vida cívica y política no son significativos y que las tecnologías digitales no incrementan la participación. Es decir, las tecnologías digitales robustecen las participaciones de quienes ya estaban comprometidos (inclusive de los antidemócratas), pero no involucran un número significativo de nuevos participantes.

Además de ciertas ambivalencias, también deben reconocerse algunos datos desilusionantes. En el 2016 el Pew Research Center constató que únicamente el $20 \%$ de los usuarios norteamericanos de los medios sociales digitales modificaron su postura política o social después de indagar en los contenidos disponibles en internet. El cambio de una quinta parte de los electores norteamericanos que se ubican en el universo de ciudadanos informados a través de internet no incrementa efectos democratizadores del sistema político, solo muestra que hay mudanza de posicionamientos dentro del mismo sistema. Menos aún hay una evolución democrática en el contexto norteamericano porque procede según la normativa con un colegio electoral, a pesar de las sospechas que puedan verificarse por la intervención de Cambridge Analytica para manipular la elección presidencial de EE. UU. en el año que reporta el estudio. A lo anterior debe sumarse que los datos empíricos recabados por el Centro de Investigación Pew (2008, 2010, 2016) muestran al 6o \% de los usuarios con el sentimiento de estar llenos de desinformación y propaganda, en el $55 \%$ de los casos internet aumenta la influencia de opiniones extremas, solo el $6 \%$ de los usuarios participan en una discusión en línea (que no ha sido convocada por el gobierno, ni tiene efectos vinculantes), el 49 \% de las conversaciones políticas en red se muestran llenas de enojo, el $53 \%$ de las audiencias se sienten con menos respeto y el $51 \%$ opina que es menos probable llegar a soluciones. Además, pocos son los ciudadanos que visitan sitios heterogéneos 
de internet durante un proceso electoral y las visitas tienen en promedio de cinco a diez minutos.

Otros datos desilusionantes son reportados por las investigaciones de Fuchs (2014), quien obtuvo los siguientes resultados: el 69,5\% de los ciudadanos consideran que utilizar tecnologías digitales como Facebook, Twitter y Youtube es benéfico, pues los activistas pueden entablar vínculos con el público y los interesados en las causas de los movimientos. Sin embargo, el $55,9 \%$ sospechan una vigilancia estatal y corporativa de los activistas en la red y ello representa una enorme desventaja para la libertad y la democracia. Además, las peticiones electrónicas del activismo no tienen relaciones jurídicas vinculantes con las decisiones parlamentarias o del poder ejecutivo.

En resumen, puede advertirse que el uso de las tecnologías digitales no ha consolidado regímenes políticos como satisfactoriamente democráticos.

\section{CONCLUSIÓN}

En primer lugar, se patentiza que las prácticas democráticas llevadas a cabo con las tecnologías digitales son de diversa índole y no siempre alcanzan el mismo nivel de relevancia. Por lo tanto, cabe suscribir lo que Schudson ha mostrado: «Internet puede hacer unas cosas por la democracia, pero otras no» (2006: 14). Entre las cosas que hace a favor están: ampliación de la esfera pública para la participación ciudadana; colaboración con instituciones u organizaciones tradicionales por medio de transacciones sociales; apoyo a campañas, organización de asambleas; organización de estructuras colaborativas que promueven la gobernanza; amplificación de elementos democráticos ya existentes en el marco jurídico... Sin embargo, las tecnologías digitales no pueden construir toda la democracia porque esta tiene elementos que implican el funcionamiento de diferentes agentes, ninguno de ellos determina de forma aislada el funcionamiento democrático y las tecnologías digitales no inciden por igual en todos ellos (García, 2016: 172).

Por lo tanto, los aportes de las tecnologías no pueden homogeneizarse en la democracia; tanto porque existen muchos modelos teóricos de la misma, como por las distintas circunstancias (históricas, de tradición, educación o identidad cultural, estipulaciones jurídicas, etc.) que requieren diversas modalidades para su ejecución. Es decir, resulta impensable que exista un único modelo universal de democracia ejecutable para todos los intentos de organi- 
zación del poder público (Saward, 2021). Por ende, los aportes significativos de las tecnologías digitales resultan circunscritos a proyectos particulares (Vedel, 2003: 230).

En segundo lugar, es notorio que cuando las tecnologías digitales amplifican las fuerzas existentes, también lo hacen a favor del poder que se resiste a los procesos democráticos o para perpetuar las prácticas antidemocráticas. Las tecnologías digitales fortalecen las acciones que ya estaban presentes, tanto del lado democratizador como del contrario. Por tanto, también son catalizadores de las brechas políticas.

En tercer lugar, las tecnologías digitales han traído nuevos problemas endógenos al maximizar riesgos de índole ética: cámaras eco, violación de la privacidad, control con algoritmos, etc. Por ende, se requieren diseños autocorrectivos de las dificultades que a su vez provocan. En este sentido, el creador de la red insiste en que debe existir equitativa regulación hacia los gobiernos y las empresas de servicios digitales (Berners-Lee, 2019). Ello lleva a destacar el desafío político por encima del tecnológico. Y por encima del desafío político está el reto ético, como lo indican Innerarity y Colomina en el contexto de la democracia algorítmica: «La revolución digital no necesita censura, necesita ética» (Innerarity y Colomina, 2020: 21).

Respecto al debilitamiento de las mediaciones, las prácticas comunicativas de las tecnologías digitales se han caracterizado más por la emisión que por la recepción, pues, si bien no eliminan definitivamente a los intermediarios o representantes tradicionales, buscan reemplazarlos. En pocas palabras, cuando el ciudadano común se dirige a las autoridades a través de las tecnologías digitales, ignorando las vías institucionalizadas, se enfrenta a dos posibles fatalidades: no tiene garantías de ser escuchado y corre el riesgo de marginar a las instituciones que tienen la misión comunicativa, representativa o de defensa de sus derechos políticos.

Un desafío político que corresponde al uso omiso de la inteligencia artificial es el de implementar métodos de votación que buscan fórmulas democráticas de selección de los mejores candidatos por ponderación, el cambio del voto en ciclos o ajuste a las tendencias de la primera intención de voto a medida que se van conociendo los resultados, la representación proporcional más justa, la votación suplementaria, la defensa de las preferencias minoritarias, etc. (métodos como D'Hondt, Dodgson, Schulze, Borda, Condorcet, Copeland, por enunciar los más conocidos). Las tecnologías digitales no han sido utilizadas para extender su aplicación y dotarlos de mayor transparencia, en la 
mayoría de los casos se han limitado a procesos de elección por mayoría simple. Tampoco se han desarrollado para influir en la evolución del marco jurídico que regula las participaciones políticas o instancias de la democracia electrónica. En este sentido, parece que existen dos grupos que no pueden formar una comunidad, aunque ejerzan mutua influencia entre sí: los investigadores de la democracia y los desarrolladores de inteligencia artificial (Paulin, 2020: 462-463).

En la práctica democrática, el desarrollo de la inteligencia artificial es extraño para los ciudadanos sin capacitación en tecnología digital y también para los gobernantes con resistencia a las innovaciones tecnológicas. Por ende, resulta falsa la pretendida reducción de costos que aporta la tecnología a la política, sobre todo en la etapa de transición en que se ejerce la participación simultáneamente de manera presencial y digital, sea por razones generacionales, por pruebas piloto o por ensayos de innovación.

Los mecanismos digitalizados de participación ciudadana (voto, presupuestos participativos, iniciativas legislativas y peticiones, plebiscitos, etc. de índole electrónica), debido a la inseguridad cibernética y a la vigilancia gubernamental, despiertan desconfianza. La complejidad inherente a la especialización tecnológica de softwares y del hardware no presenta a los instrumentos de participación política digitalizados como deseables, fiables y auditables ante el ciudadano común. Los bloques de cadenas de seguridad, sumados a los códigos con criptografía homomórfica basada en curvas elípticas supersingulares, no son del habitual entendimiento de los participantes. Cuando las condiciones tecnológicas de control (encriptaciones, transmisiones cifradas, cómputo y recepción verificativa por códigos temporales, etc.) se incrementan para garantizar mayor seguridad y evitar fraudes o delitos, entonces generan brechas tecnológicas y dificultades para que los ciudadanos legos participen. Además, el uso de códigos abiertos o de propiedad privada de los programas digitales detona polémicas sobre el alcance de derechos de autor, control y costos.

La participación ciudadana digitalizada, en la medida que puede ejercerse telemáticamente, es decir, a distancia y sin la protección presencial de la autoridad (en jornadas electorales, asambleas, localidades para la consulta ciudadana, etc.), también presentan el riesgo de ser sometidos a coacción o amenazas. En el peor de los casos, a la mercantilización de compra de votos, opiniones en las consultas o preferencias en referéndums, peticiones y revocatorias. 
Desde los desafíos políticos y éticos, la tarea de las tecnologías digitales a futuro apunta a utilizar los desarrollos de la inteligencia artificial para instrumentar el mayor número posible de mecanismos de participación ciudadana, ampliar su alcance demográfico para abatir las brechas, construir diseños autocorrectivos ante sus problemas endógenos, complementar los procedimientos de mediación y promover la deliberación incluyente, mientras que el futuro de la democracia electrónica está en la pedagogía tecnopolítica: comprender y controlar los entramados internos de las tecnologías digitales, anticiparse a los riesgos éticos con la autoorganización ciudadana para que la distribución y equilibrio del poder político alcance equitativas oportunidades de creación colectiva en las decisiones públicas.

\section{BIBLIOGRAFÍA}

Abramson, Mark y Means, Grady (2001). e-Government 2001. Lanham: Rowman and Littlefield Publishers.

Aguirre, Jorge y Jones, Mark (2012). The Use of Electronic Technology and Legislative Representation in the Mexican and U.S. States: Nuevo León and Texas. USA: Puentes Consortium Project.

Anduiza, Eva, Jensen, Michael y Jorba, Laia (2012). Digital Media and Political Engagement Worldwide. A Comparative Study. Cambridge: Cambridge University Press.

Barber, Benjamín (2006). ¿Hasta qué punto son democráticas las nuevas tecnologías de telecomunicación? IDP Revista de Internet, Derecho y Política, 3, 17-27.

Bellamy, Christine (2000). Modelling electronic democracy: towards democratic discourses for an information age. En Hoff, Jens, Horrocks, Ivan y Tops, Pieter (Eds.) Democratic Governance and New Technology (34-55). London: Routledge. doi: 10.4324/9780203449738_chapter_2

Bellin, Eva (2012). Reconsidering the robustness of authoritarianism in the Middle East: Lessons from the Arab Spring. Comparative Politics, 44(2), 127-149. 
Berners-Lee, Tim (2019). Contract for the web. Recuperado de: https://contractfortheweb.org/es/ [Consultado el 23 de marzo de 2021].

Boulianne, Shelley (2015). Social media use and participation: A metaanalysis of current research. Information, Communication \& Society, 18(5), 524-538. doi: 10.1080/1369118X.2015.1008542

Boutyline, Andrei y Willer, Robb (2017). The social structure of political echo chambers: Variation in ideological homophily in online networks. Political Psychology, 38(3), 551-569. doi: 10.1111/pops.12337

Calvo, Patrici (2019a). Democracia algorítmica: consideraciones éticas sobre la dataficación de la esfera pública. Revista del CLAD Reforma y Democracia, 74, 5-30.

Calvo, Patrici (2019b). Etificación, la transformación digital de lo moral. Kriterion, 144, 671-688. doi: 10.159o/0100-512X2019n14409pc

Castells, Manuel (2001). La Galaxia Internet. España: Plaza y Janés.

Castells, Manuel (2009). Comunicación y poder. Madrid: Alianza Editorial.

Cha, Meeyoung, Haddadi, Hamed, Benevenuto, Fabrício y Gummad, Krishna (2010). Measuring User Influence on Twitter: the Million Follower Fallacy. En Hearst, Marti (Ed.). Proceedings of the Fourth International AAAI Conference on Weblogs and Social Media (7-10). Washington: AAAI Press.

Chadwick, Andrew (2006). Internet Politics. Oxford: Oxford University Press.

Chadwick, Andrew y Howard, Philip (Eds.) (2009). The Routledge Handbook of Internet Politics. New York: Routledge.

Caldevilla, David (2009). Democracia 2.0: La política se introduce en las redes sociales. Pensar la publicidad, 3(2), 31-48

Comité de Ministros del Consejo de Europa (2009). Recomendación CM / Rec (2009) 1 del Comité de Ministros a los Estados miembros sobre la democracia electrónica (e-democracia). Recuperado de: http://documentostics.com/component/option,com_docman/task, 
doc_download/gid,1495/Itemid,3/ [Consultado el 23 de marzo de 2021].

Cotarelo, Ramón (2002). ¿Democracia electrónica vs. Democracia deliberativa? En Cairo Carou, Heriberto (Comp.). Democracia digital, límites y oportunidades (9-12). Madrid: Trotta.

Flaxman, Seth, Goel, Sharad y Rao, Justin (2016). Filter bubbles, echo chambers, and online news consumption. Public Opinion Quarterly, 8o(S1), 298-320. doi: 10.1093/poq/nfwoo6

Fuchs, Christian (2014). Retos para la democracia: Medios sociales y esfera pública. Telos, 98, 71-82.

García, Elena (2016). Democracia digital. Discursos sobre participación ciudadana y TIC. Revista de Estudios Políticos, 173, 169-193. doi: $10.18042 /$ cepc/rep.173.05

Hagen, Martin (1997). A Typology of Electronic Democracy. Hamburg: Ms.

Hennen, Leonhard, Keulen, Ira Van, Korthagen, Iris, Aichholzer, Georg, Lindner, Ralf, Nielsen y Rasmus Øjvind (2020). European E-Democracy in Practice. Germany: Springer.

Hindman, Matthew (2009). The Myth of Digital Democracy. New Jersey: Princeton University Press.

Howard, Philip, Savage, Saip, Flores, Claudia, Toxtli, Carlos y Monroy-Hernández, Andres (2016). Social Media, Civic Engagement, and the Slacktivism Hypothesis: Lessons from Mexico's El Bronco. Journal of International Affairs, 70(1), 55-73.

Innerarity, Daniel (2015). Desenredar una ilusión: notas para una teoría crítica de la democracia digital. En Champeau, Serge y Innerarity, Daniel (Comps.) (37-43). Internet y el futuro de la democracia. Barcelona: Paidós.

Innerarity, Daniel y Colomina, Carme (2020). La verdad en las democracias algorítmicas. Revista CIDOB d'Afers Internacionals, 124, 11-23. doi: 10.24241/rcai.2020.124.1.11 
Keen, Andrew (2012). Digital Vertigo: How Today's Online Social Revolution Is Dividing, Diminishing, and Disorienting Us. New York: St. Martin's Press.

Kosinski, Michal. (2017). Big data, inteligencia artificial y el futuro de la democracia. En Kosinski, Michal (Ed.), Aportes al debate parlamentario. México: Senado de la República.

Krimmer, Robert (2009). The map Modern democracy. The Modern Democracy Magazine, 1, 8-9.

Levitsky, Steven y Ahmad, Lucan (2015). The Myth of Democratic Recession. Journal of Democracy, 26(1), 45-58.

Lessig, Lawrence (1999). Code: And Other Laws of Cyberspace. New York: Basic Books, Inc.

Lidén, Gustav (2012). Is e-democracy more than democratic? - An examination of the implementation of socially sustainable values in e-democratic processes. Electronic Journal of e-Government EJEG, 10(1), 84-94.

Lindner, Ralf, Beckert, Bernd, Aichholzer, Georg, Strauß, Stefan y Hennen, Leonhard (2010). E-democracy in Europe - prospects of internet-based political participation. Interim Report - Phase I. Brussels/Strasbourg: European Parliament, Science and Technology Options Assessment STOA.

Lindner, Ralf y Aichholzer, Georg (2020). E-Democracy: Conceptual Foundations and Recent Trends. En Henne, Leonhard, van Keulen, Ira, Korthagen, Iris, Aichholzer, Georg, Lindner, Ralf y Øjvind Nielsen, Rasmus (Eds.). European E-Democracy in Practice (11-45). Germany: Springer.

Morozov, Evgeny (2011). The net delusion: The dark side of Internet freedom. New York: Public Affairs.

Moss, Giles y Coleman, Stephen (2014). Deliberative Manoeuvres in the Digital Darkness: e-Democracy Policy in the United Kingdom. British Journal of Politics and International Relations, 16, 410-427. doi: 10.1111/1467-856X.12004 
Päivärinta, Tero y Saebo, Øystein (2006). Models of E-democracy. Communications of the Association for Information Systems, 17, 818840. doi: 10.17705/1CAIS.01737

Pariser, Eli y Helsper, Ellen (2011). The Filter Bubble. What the Internet is hiding from you. London: Penguin.

Paulin, Alois (2020). Ten years of liquid democracy research: An overview. Central and Eastern European EDem and EGov Days, 338, 455-466. doi: 10.24989/ocg.338.36

PEW Research Center (2008). The Internet and the 2008 Election. Recuperado de: http://www.pewinternet.org/2008/06/15/theinternet-and-the-20o8-election/ [Consultado el 23 de marzo de 2021].

PEW Research Center (2010). The internet and campaign 2010 survey. Recuperado de: http://www.pewinternet.org/ /media/Files/Repor ts/2011/Internet\%20and\%20 Campaign\%202010.pdf. [Consultado el 23 de marzo de 2021].

PEW Research Center (2016). The political environment on social media. Recuperado de: PI_2016.10.25_Politics-and-SocialMedia_FINAL.pdf. [Consultado el 23 de marzo de 2021].

Porrúa, Miguel (2004). Gobierno electrónico: conceptos y avances. Revista del CLAD Reforma y Democracia, 3o, 1-11.

Rheingold, Howard (1993). The Virtual Community: homesteading on the electronic frontier. New York: Harper Collins

Ricci, Andrea (2013). The Early Political Web (1995-2005): A ten-year observational research seeking evidence of eDemocracy in the information architecture of political parties' web sites worldwide. Thèse de Docteur. Belgium: Université Libre de Bruxelles.

Runciman, David (2017). Political Theory and Real Politics in the Age of the Internet, The Journal of Political Philosophy, 25(1), 3-21. doi: 10.1111/jopp. 12087

Saward, Michael (2021). Democratic Design. Oxford: Oxford University Press. 
Schudson, Michael (2006). New technologies and Not-So-New Democracies. MedieKultur Journal of media and communication research, $22(40), 1-9$.

Simon, Julie, Bass, Theo, Boelman, Victoria y Mulgan, Geoff (NESTA) (2017). Digital Democracy. The tools transforming political engagement. London: NESTA \& MacArthur Foundation Research Network on Opening Governance.

Smyrnaios, Nikos (2016). L'effet GAFAm: stratégies et logiques de l'oligopole de l'internet. Communication \& langages, 188(2), 61-83. doi: 10.4074/So336150016012047

Trechsel, Alexander H., Kies, Raphael, Mendez, Fernando y Schmitter, Philippe C. (2004). Evaluation of the use of new technologies in order to facilitate democracy in Europe. Luxemburg: European Communities.

Van Alstyne, Marshall y Brynjolfsson, Erik (2005). Global village or cyber-balkans? Modeling and measuring the integration of electronic communities. Management Science, 51(6), 851-868. doi: $10.1287 /$ mnsc. 1050.0363

Van Dijk, Jan y Hacker, Kenneth (200o). Digital Democracy: Issues of Theory and Practice. New York: Sage Publications.

Van Dijk, Jan y Hacker, Kenneth (2018). Internet and Democracy in the Network Society. New York: Routledge.

Vedel, Thierry (2003). Lidée de démocratie électronique. Origines, visions, questions. En Perrineau, Pascal (Dir.). Le désenchantement démocratique (243-266). La Tour d'Aigues: Editions de l'Aube.

Wright, Rosemary y Jacobs, Jerry (1994). Male Flight from Computer Work: A New Look at Occupational Resegregation and Ghettoization. American Sociological Review, 59(4), 511-536. doi: $10.2307 / 2095929$

Zuboff, Shoshana (2019). The Age of Surveillance Capitalism: The Fight for a Human Future at the New Frontier of Power. New York: Public Affairs. 